\title{
OBJECT RECOGNITION BASED ON LBP AND DISCRETE WAVELET TRANSFORM
}

\author{
Jayasudha A, \\ Department of Computer Science, \\ SRM Arts and Science College, \\ Kattankulathur, Chennai, India \\ jsudhaannamalai@gmail.com \\ Priya K, \\ Department of Computer Science, \\ SRM Arts and Science College, \\ Kattankulathur, Chennai, India \\ kpriyaa@gmail.com
}

\begin{abstract}
Automated object recognition from images plays a significant role in many computer vision systems such as content based image retrieval, navigation of robots and object manipulation processes. In this paper, Local Binary Patterns (LBP) and Discrete Wavelet Transform (DWT) techniques are analyzed for object recognition. The later technique helps us to extract the detailed information's of objects from its multi-scale representation. Using the extracted features, the recognition of objects can be done by the classifier known as the nearest neighbour classifier. A maximum of $98.03 \%$ average recognition accuracy is attained by the system with Columbia Object Image Library (COIL-100) objects while using the features of LBP and 6th level DWT energies.
\end{abstract}

Keywords: LBP, Nearest Neighbour Classifier, Wavelet Transform, Object Recognition.

\section{INTRODUCTION}

Object recognition by computers is very difficult task. Many computer vision approaches are developed recently to detect or classify objects in a scene. The inter-class correlation and intra-class diversity is analyzed for object recognition in [1]. These parameters are obtained from a group of sensitive multiple kernel learning method. The performance is increased with a hybrid grouping strategy.

Colour descriptors are used for object recognition in [2]. They are extracted from a distinct region which covers multiple segments of given object image. These regions are identified by edge mapping and clustering. A prediction method for fundamental presentation of objects is explained in [3]. It also considers the various distortion factors such as occlusion, insecurity, and clutter.

An object recognition process discussed in [4] makes use of a multi-linear supervised neighbourhood model to extract the features. In order to represent an image, a local descriptor tensor is used for subject recognition. By the sparse function representation, the approximation of spatial dimensions together with orientation dimension is discussed in [5] for object recognition. It can develop a rich amount of absolute information about the object views.

An approach for part based object recognition is described in [6]. It is achieved by the collection of correlograms of images that represents specific attributes at the same time in several images. An approach for object recognition 
using empirical wavelet transform for the extraction of the energy and entropy features are discussed in $[7,8]$.

Kernel dictionary learning based object recognition is discussed in [9]. The prototype model is continuously optimized in order to get promising results. A method based on hidden Markov models is described in [10]. It can recognize occlude objects. For each class, a hidden Markov model is created and trained with ensembles.

In this paper, an approach to classify objects is presented. Both spatial and frequency domain features are extracted from LBP and wavelet respectively. It does not consider the occlusion. Section 2 gives the overview of methods and materials for object recognition system followed by the results and discussion section. Finally, the last section concludes the object recognition system.

\section{METHODS AND MATERIALS}

The method for object recognition is built based on feature extraction process by LBP and DWT at first. Then, these features are tested by the classifier where the output is obtained in the second stage. The framework of object recognition system by LBP+DWT is shown in Figure 1 .

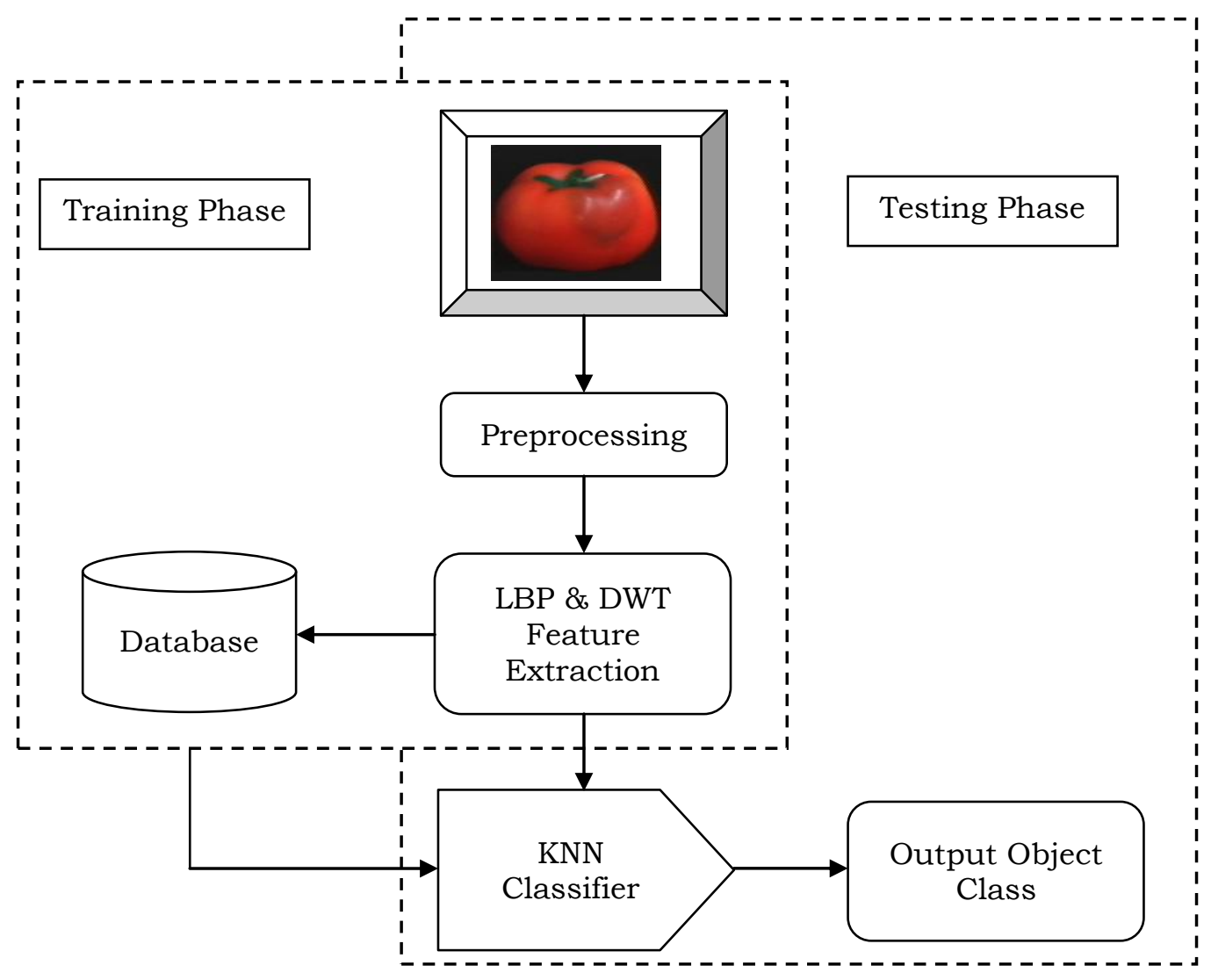

Fig. 1 LBP and DWT based object recognition system 
In this study, only the gray information is considered for feature extraction process. To get the gray information, Eqn. 1 is applied to the given images in RGB mode.

$$
G R A Y=0.2989 R+0.5870 G+0.1140 B
$$

where $\mathrm{B}, \mathrm{R}$, and $\mathrm{G}$ are the blue, red, and green colour components. Then, LBP features are computed by using the following Eqn.

$$
\operatorname{LBP}\left(X_{c}, Y_{c}\right)=\sum_{n=0}^{7} 2^{n} S\left(i_{n}-i_{c}\right)
$$

where $i_{c}$ and $i_{n}$ indicate the value of central pixel and $N$ neighbourhood pixels. Figure 2 shows the computation of LBP operator on eight neighbourhood pixels.

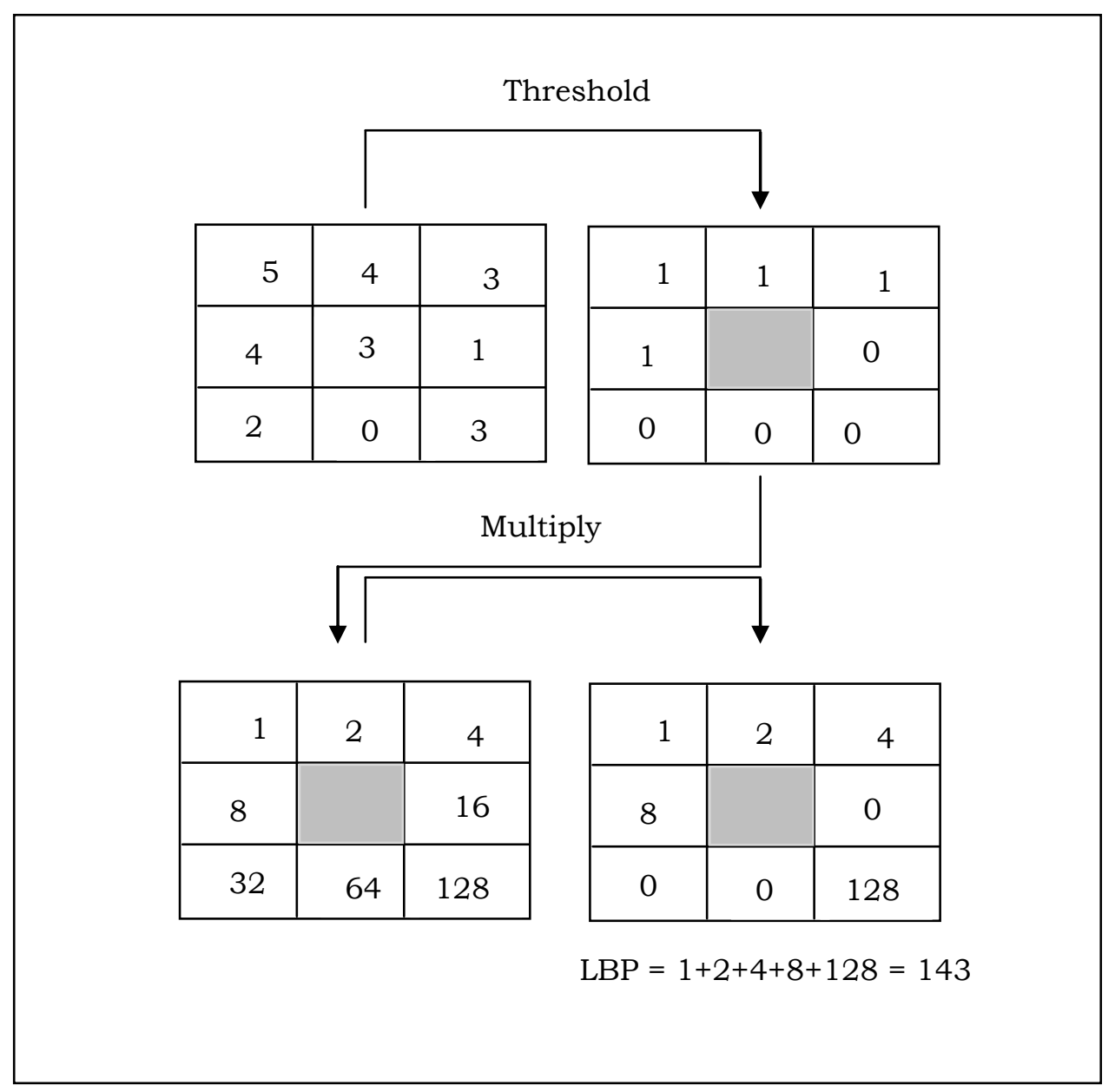

Fig. 2 Computation of LBP operator

The application of the process in Figure 2 produces a scalar quantity for every $3 \times 3$ matrix. For the whole image, a number of LBP are obtained, and a histogram of LBPs is computed. A sample image is shown in Figure 3 (a) and its corresponding LBP histogram is shown in Figure 3 (b). 


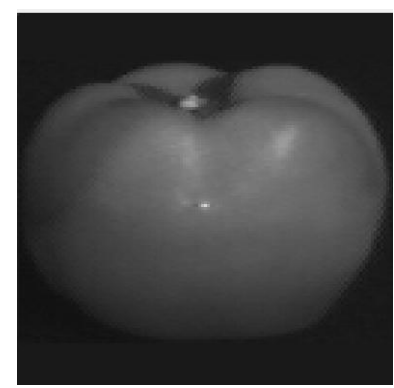

(a)

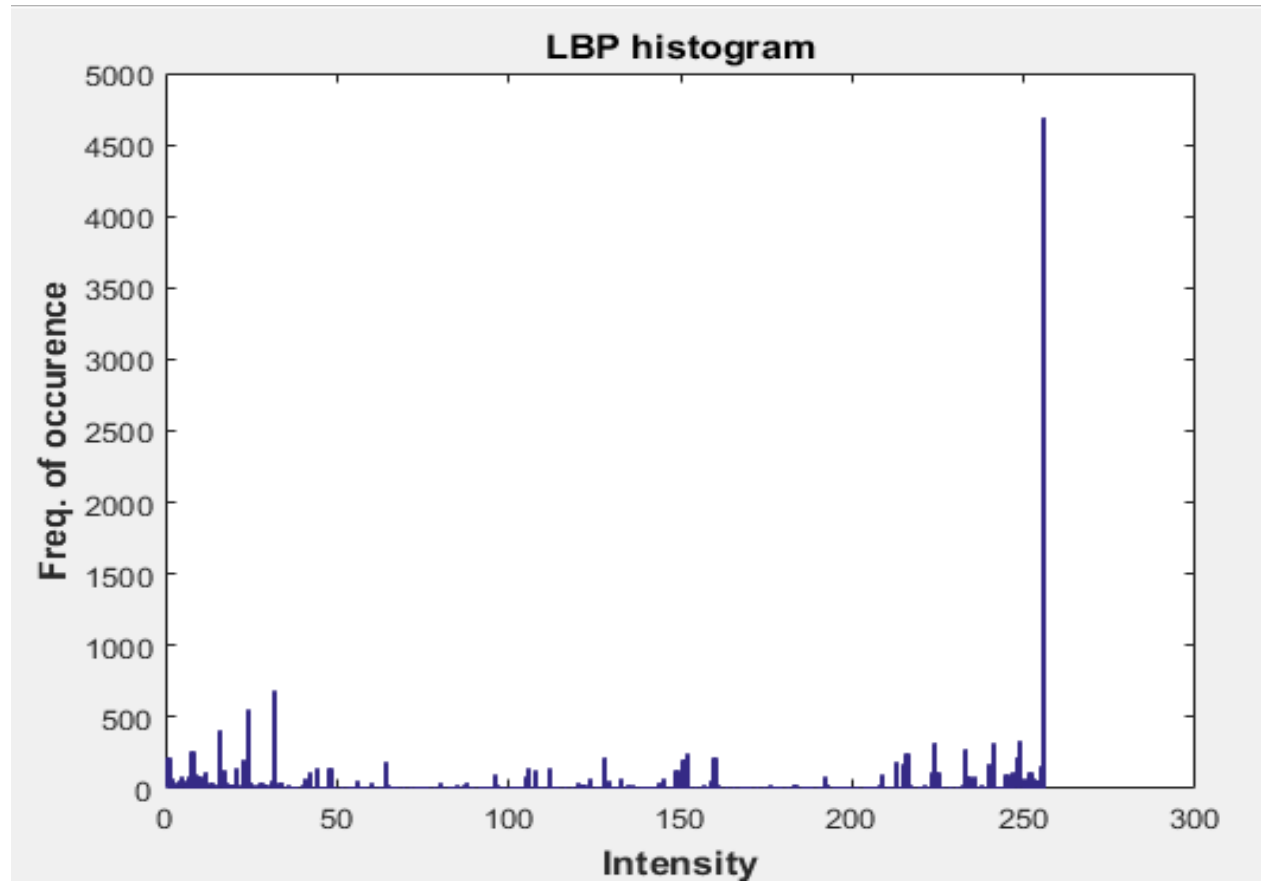

(b)

Fig. 3 (a) Input image (b) LBP histogram

Along with this histogram, DWT based features are combined to form a better representation of given object. As the lower frequency sub-bands approximate the given object, only the detailed information's from all highfrequency sub-bands are utilized. This process is repeated to get features all training objects in the database with their labels. In this work, a simple nearest neighbour classifier is used to recognize the given test object by providing testing features with the training database.

\section{RESULTS AND DISCUSSION}

The recognition system using LBP and DWT features are tested with COIL100 object database [11]. It consists of 100 different objects with a resolution of 128 x128 pixels. All are colour images with 8-bit depth. The different objects in the COIL database are shown in Figure 4. Each object has 72 images captured at 5 degrees of rotation. 


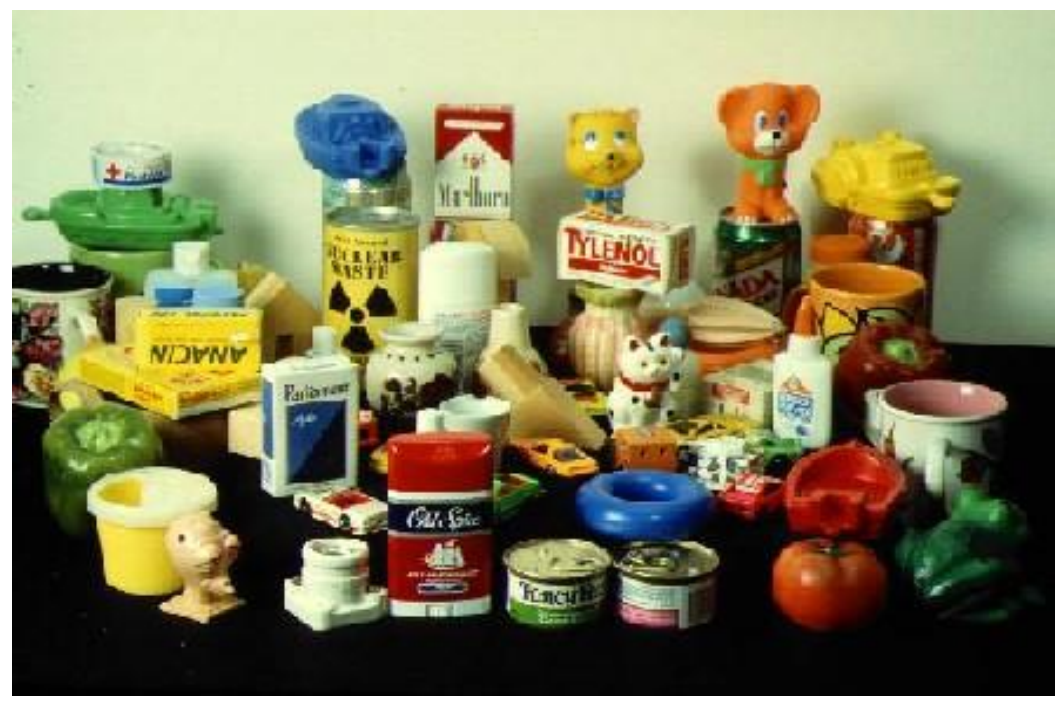

Fig. 4 COIL Database

Based on the angle of rotations, the sample images of each COIL-100 object are separated for training the classifier. The accuracies obtained by the system using the training images of different degree of rotations such as 10,20 , $30,45,60$, and 90 are shown in Table 1. Figure 5 shows the different rotations of a single object in the database.
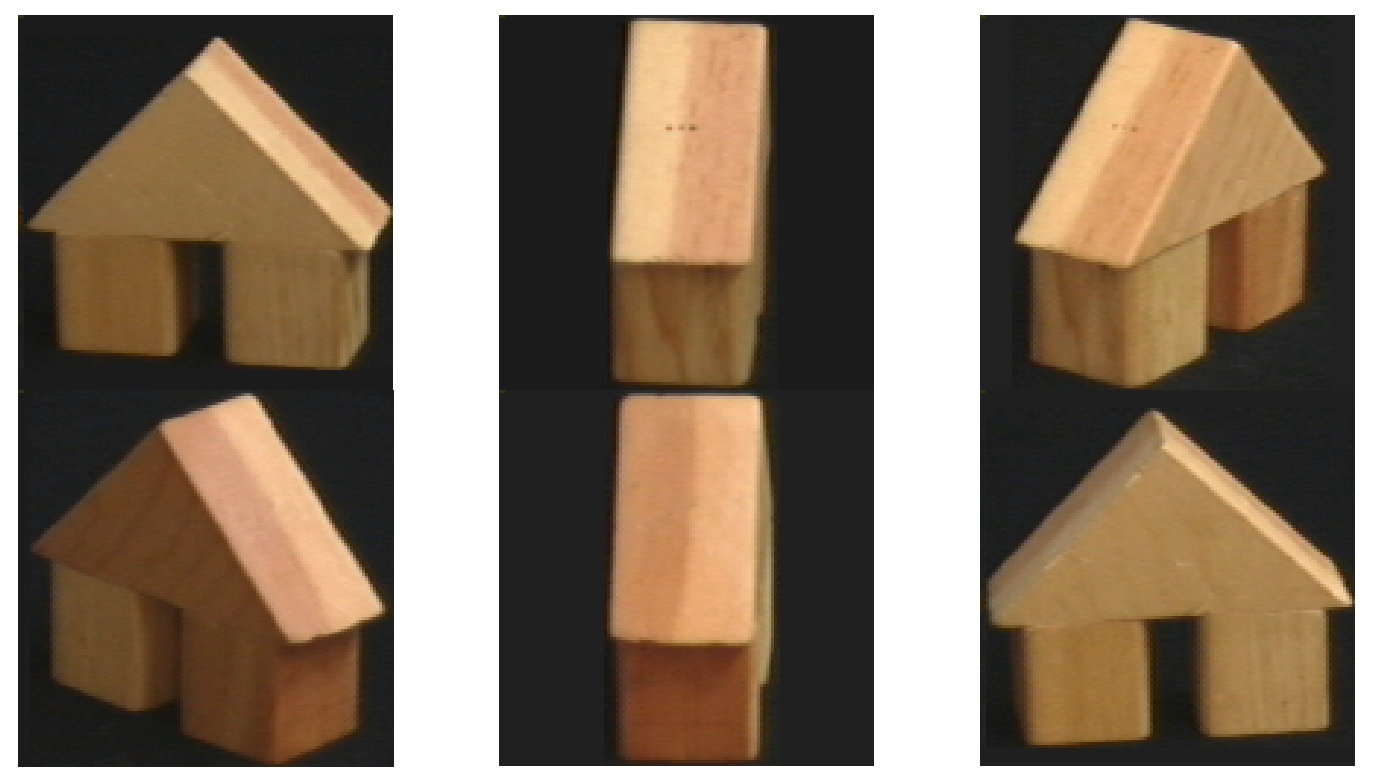

Fig. 5 Different Rotations of a sample image in the COIL Database

For analyzing the system, 2 to 6 level of DWT decomposition are used. For classification, the nearest neighbour makes use of Euclidean distance as a distance measure. Average recognition accuracies obtained by the system are shown in Table 1 for different DWT decomposition levels. 
TABLE 1 Average Recognition accuracies by $L B P+D W T+K N N$

\begin{tabular}{|c|c|c|c|c|c|c|}
\hline \multirow{2}{*}{$\begin{array}{c}\text { Level of } \\
\text { Decomposition }\end{array}$} & \multicolumn{7}{|c|}{ Recognition accuracy (\%) } \\
\cline { 2 - 7 } & $\mathbf{1 0}^{\mathbf{0}}$ & $\mathbf{2 0}^{\mathbf{0}}$ & $\mathbf{3 0}^{\mathbf{0}}$ & $\mathbf{4 5}^{\mathbf{0}}$ & $\mathbf{6 0}^{\mathbf{0}}$ & $\mathbf{9 0}^{\mathbf{0}}$ \\
\hline 2 & 86.25 & 78.57 & 73.18 & 66.42 & 60.04 & 52.51 \\
\hline 3 & 86.38 & 77.80 & 72.02 & 65.84 & 57.03 & 50.71 \\
\hline 4 & 88.91 & 80.72 & 76.15 & 69.45 & 63.17 & 57.63 \\
\hline 5 & 94.52 & 88.31 & 82.30 & 74.03 & 67.76 & 60.51 \\
\hline 6 & 98.03 & 93.57 & 88.85 & 82.63 & 75.95 & 68.57 \\
\hline
\end{tabular}

It is observed from the Table 1 that while reducing the number of training images reduces the average recognition accuracy of the system. Every 10 degree of rotation objects in the training set produces a better accuracy of $98.03 \%$ at 6 th level DWT and LBP features.

\section{CONCLUSION}

In this paper, LBP and DWT based features with nearest neighbour classifier are analyzed for object recognition automatically. The objects in COIL100 are represented by the sub-band energies of DWT along with LBP features. Many simulations are done by changing training objects which are captured in different angles. A maximum of $98.03 \%$ average recognition accuracy of 100 COIL database objects is attained by the system while using LBP and 6th level DWT energy features. In future research, the colour information can also be used to improve the system performance.

\section{REFERENCES}

[1]. J. Yang, Y. Tian, L.Y. Duan, T. Huang, and W. Gao, "Group-sensitive multiple kernel learning for object recognition", IEEE Transactions on Image Processing, Vol. 21, No. 5, 2012, pp. 2838-2852.

[2]. S.K. Naik, and C.A. Murthy, "Distinct multicolored region descriptors for object recognition", IEEE transactions on pattern analysis and machine intelligence, Vol. 29, No. 7, 2007, pp. 1291-1296.

[3]. M. Boshra, and B. Bhanu, "Predicting performance of object recognition", IEEE Transactions on Pattern Analysis and Machine Intelligence, Vol. 22, No. 9, 2000, pp. 956-969.

[4]. X.H. Han, Y.W. Chen, and X. Ruan, "Multilinear supervised neighborhood embedding of a local descriptor tensor for scene/object recognition", IEEE Transactions on Image Processing, Vol. 21, No. 3, 2012, pp. 1314-1326.

[5]. T.V. Pham, and A.W. Smeulders, "Sparse representation for coarse and fine object recognition", IEEE transactions on pattern analysis and machine intelligence, Vol. 28, No. 4, 2006, pp. 555-567.

[6]. J. Amores, N. Sebe, and P. Radeva, "Context-based object-class recognition and retrieval by generalized correlograms", IEEE Transactions on Pattern Analysis and Machine Intelligence, Vol. 29, No. 10, 2007, pp. 1-34.

[7]. S. Murugan, B. Anjali, and T.R. Ganeshbabu, "An efficient approach for object recognition using empirical wavelet transform", International Journal of Modern Sciences and Engineering Technology, Vol. 3, No. 11, 2016, pp.34-39.

[8]. S. Murugan, B. Anjali, and T.R. Ganeshbabu, "Object recognition based on empirical wavelet transform", International Journal of MC Square Scientific Research, Vol. 7, No. 1, 2015, pp. 77-83. 
[9]. H. Liu, and F. Sun, "Online kernel dictionary learning for object recognition", IEEE International Conference on Automation Science and Engineering, 2016, pp. 268-273.

[10]. F.A. Guerrero-Pena, and G.C. Vasconcelos, "Search-Space Sorting with Hidden Markov Models for Occluded Object Recognition", IEEE $8^{\text {th }}$ International Conference on Intelligent Systems, 2016, pp. 47-52.

[11]. S.A. Nene, S.K. Nayar, and H. Murase, "Columbia Object Image Library (COIL-100)", Technical Report CUCS-006-96, 1996. 